\title{
In Situ Drug Release Monitoring with a Fiber-Optic System: Overcoming Matrix Interferences Using Derivative Spectrophotometry
}

\author{
Alexis Guillot ${ }^{1,2}$, Markus Limberger ${ }^{1}$, Johannes Krämer ${ }^{1, *}$, \\ and Claus-Michael Lehr ${ }^{2}$ \\ 'PHAST GmbH, Kardinal-Wendel-Str. 16, D-66424 Homburg, Germany \\ ${ }^{2}$ Helmholtz Institute for Pharmaceutical Research Saarland (HIPS), Helmholtz Center for Infection Research (HZI), Department DDEL, \\ Saarland University, Campus A41, D-66123 Saarbrücken, Germany
}

\begin{abstract}
The use of automation has increased in all the fields of the pharmaceutical industry, such as manufacturing and quality control. In the field of in vitro dissolution testing, the fiber-optic system is often considered a promising tool because it allows in situ monitoring of the drug release without manual sampling, liquid transfer, and hence phase separation. This paper evaluates the use of fiber-optic in vitro dissolution testing and presents a technique to compensate for analytical interferences caused by excipients.
\end{abstract}

\section{INTRODUCTION}

$$
\text { I }
$$

o assess product performance and the quality of pharmaceuticals, in vitro dissolution tests are regularly carried out in the pharmaceutical industry. Over the past decades, these tests were more commonly performed manually. The tendency in industry is to reduce random error and optimize time and cost by automation. Dissolution testing is a composite procedure, with the bath as a sample preparation device, sampling and other transfer actions for liquids, filtration to obtain the portion dissolved, chemical analysis (HPLC, spectrophotometry), and data processing at the end. All of the steps may introduce additional error, decreasing the precision of the final results. Automated systems may include automated sampling, processing of sample solutions, online quantification, and even cleaning. However, to reduce the number of steps to both accelerate the process and increase the precision of dissolution methods, alternative solutions may allow omission of the sample and filtration steps by measuring drug release directly in the vessels. Suitable probes that are connected by glass fiber to the spectrophotometer enable UV measurements at any selected time point. The goal of this paper is to review the suitability of fiber-optic systems for dissolution testing and to describe the limitations for use. An example of a fiber-optic system with a "rod" probe is provided to expand the use. A method to compensate for the interferences by undissolved particles during in situ monitoring of the drug release is presented.

\section{DESCRIPTION OF THE SYSTEM USED FOR THE EXPERIMENTS}

The system comprised a UV-vis spectrophotometer (Varian Cary 50 Tablet) equipped with the Cary WinUV software and connected to an Agilent dissolution apparatus $708-D S$ by fiber optics. Among the different types of probes (1), the rod type was selected (Figure 1). The complete fiber-optic system is commercially available from Agilent Technologies.

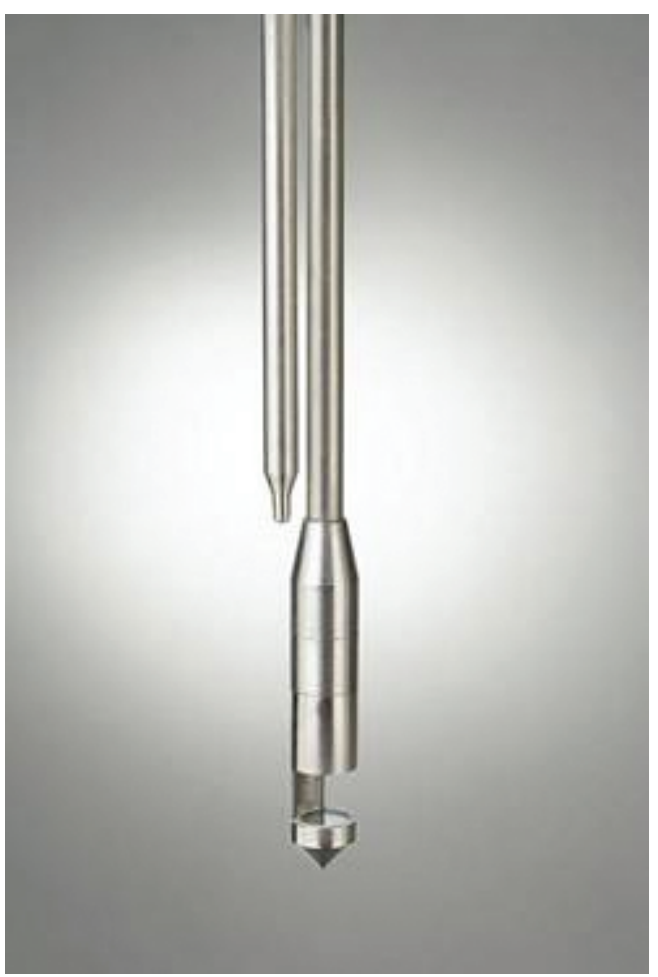

Figure 1. Temperature probe (left) with optic rod probe (right) (source: Agilent Technologies).

*Corresponding author. 


\section{Advantages}

In addition to the general advantages provided by automated systems (e.g., sparing time, workforce), fiber-optic systems for in vitro dissolution offer the following attractive features:

1) Drug dissolution is measured in situ.

Firstly, the in vitro drug release studies become more accurate and precise as they allow observations of the phenomena as they occur in the vessel. No disturbances are introduced into the system by sampling. Sampling in the sense of dissolution kinetics implies the assumption that the aliquot withdrawn is representative of the dissolution process at a given time point and that the removal has no effect on either the aliquot or the entire system (i.e., its hydrodynamics). In situ monitoring does allow recording of the dissolution kinetics exactly at the site of action. Because no sample solution is consumed, the sampling may be adapted to the grid needed to describe the kinetics adequately. The number of time points for analysis is not relevantly limited.

2) Measurements can be performed for small volumes. Possible interferences due to changes of the hydrodynamics are caused firstly by the relationship of the individual sample volume to the total volume and secondly by the total volume withdrawn as a function of sampling schedule. Fiber-optic measurements do not require sample removal. Therefore, the mechanical dissolution conditions remain unchanged throughout the experiment. The results are not biased by alterations due to volume-dependent hydrodynamics and secondary concentration changes by volume replacement with fresh medium.

3) Transfer of sample solutions is not required.

As no transfer of sample solution is required, the time of analysis is significantly shorter, in particular in parallel experimental design:

$$
\begin{array}{ll}
n=6 & \text { routine QC testing } \\
n=12 & f_{2} \text { testing } \\
n=24 & \text { USP stage testing }
\end{array}
$$

In the case of multiple sampling, the timesaving effect is relevantly greater.

4) The use of tubing, syringes, and filters is not required. The risk of API adsorption at surfaces of tubing, syringes, or filters is hence omitted. As no consumables are needed for sample processing, their validation is not needed, not to forget the cost-saving effects.

5) There are no stability issues with sample solutions. Physical instabilities as an effect of evaporation may not occur. There is no temperature gradient as for instance between the vessel $\left(37^{\circ} \mathrm{C}\right)$ and the spectrophotometric cuvette $\left(25^{\circ} \mathrm{C}\right)$. Secondary physicochemical instabilities such as temperature-dependent precipitation are avoided. As there is no time delay due to stor- age of sample solutions, chemical stability of sample solutions is not an issue.

6) The chemical analyses are in real time. In particular, in analytical method development as well as dosage form development, dissolution results are available while the test is still running. The advantage is that operating the dissolution bath and performing the chemical analysis concomitantly allows stopping experiments at any given specification or expectation time point. This advantage is valid for any online analytical coupling such as flow-through cuvette or ion-selective electrode. It is given here for completeness.

\section{Particular Caution}

In spite of the aforementioned advantages, use of a fiber-optic system requires particular caution.

1) Resident rod probes can cause turbulences in the vessel.

There are several reasons to leave the probes in the vessel for a time exceeding the pharmacopeial specifications. Among others, these are to avoid entrapment of air in the optical path and crystallization of solids on surfaces. Recording of spectra also requires longer periods. However, the resident probes may bias the dissolution results. On a drug product, level validation is required.

The interferences caused by the probes can be significantly reduced if the probes are raised between data recording from the pharmacopeial sampling point to a rest point immediately below the medium surface. As an alternative, probes may be used with hydrodynamically optimized rods. Lu et al. (1) describe alternative probes, either in the shaft of the stirrer or those adapted to the curvature of the vessel.

2) Air bubbles adhere between the mirror and the glass fiber.

Air bubbles may form by immersing the probes into the medium. Consequently, the probes should be maintained immersed all along the test. Moreover, without any efficient degassing, air bubbles may form at the probes during a test run.

3) Particles in suspension in the medium may form a thin layer on the probe.

Over the dissolution test, a partial buildup tends to occur on the mirror of the probe in the case of media containing many particles such as excipient aggregates or even undissolved API. The phenomenon can be avoided by both orienting the opening of the probe toward the walls of the vessel or the shaft of the paddle or basket and raising the manifold between the measurements.

4) Excipients may cause spectrophotometric interference. If the spectra of the API and the excipient overlap, the specificity is not given. Moreover, a simple subtraction of absorptions or the reference wavelength 
concept is generally inappropriate in the case where both the API and excipient concentrations increase over time. This may be solved by mathematical methods available to level out the interferences on the level of chemometry. For example, if the interfering compound has a maximal (or minimal) UV absorption within the absorption range of the API, then the drug substance can be quantified using the amplitude of the first derivative of the absorption spectrum at the zero-crossing point (2). Different derivative UV spectrophotometric methods have been described $(3,4)$ for interfering compounds with or without maximal, minimal, or constant absorption within the wavelength range of the API absorption. This paper focuses on implementation of the derivative technique where the interfering compound has a constant UV absorption between two wavelengths within the absorption range of the API. Currently, this method is used successfully for in vitro dissolution testing (unpublished results). For interfering light scattering, derivative (5) and other corrective methods (6) are well described.

\section{Limitations}

The two main limitations encountered for the use of fiber-optic systems are:

1) Very turbid suspensions cannot be analyzed.

In this case, the UV absorption of API is too weak compared with the total absorption of the suspension, thus quantification is impossible for very turbid suspensions.

2) Solutions with compounds producing interferences that cannot be mathematically removed.

\section{DESCRIPTION AND EVALUATION OF A DERIVATIVE SPECTROPHOTOMETRIC TECHNIQUE Theory}

For a mixture containing a given excipient and a drug substance, the resulting absorption spectrum of the mixture (D0) is the sum of the absorption for both substances:

$$
A_{\text {MiX }}(\lambda)=A_{A P I}(\lambda)+A_{E X C}(\lambda)
$$

Supposing that the UV absorption of the excipient is constant over a given wavelength range, $A_{E x C}(\lambda)=k \forall \lambda \in\left[\lambda_{1} ; \lambda_{2}\right]$, then the first derivative (D1) of the absorption spectrum of such an excipient will be close to zero within this range, further named "derivative range":

$$
\frac{d A_{E x c}(\lambda)}{d \lambda} \approx 0 \quad \forall \lambda \in\left[\lambda_{1} ; \lambda_{2}\right]
$$

Consequently, the first derivative clears up the component brought by the drug substance by neutralization of the component brought by the excipient regardless of its concentration:

$$
\frac{d A_{M i x}(\lambda)}{d \lambda}=\frac{d A_{A P I}(\lambda)}{d \lambda}+\frac{d A_{E x C}(\lambda)}{d \lambda}=\frac{d A_{A P I}(\lambda)}{d \lambda} \forall \lambda \in\left[\lambda_{1} ; \lambda_{2}\right]
$$

According to the Beer-Lambert law

$$
\begin{gathered}
A(\lambda)=\varepsilon(\lambda) \cdot C \cdot / \text { thus } \\
\frac{d A(\lambda)}{d \lambda}=\frac{d \varepsilon(\lambda)}{d \lambda} \cdot C \cdot /
\end{gathered}
$$

The API can be quantified directly using the amplitude of $\mathrm{D} 1$ within the derivative range:

$$
C \propto \frac{d A(\lambda)}{d \lambda} \forall \lambda \in\left[\lambda_{1} ; \lambda_{2}\right]
$$

However, the sensitivity may be decreased after the derivative operation, which is why the area under the curve (AUC) may be computed between two wavelengths within the derivative range.

$$
\begin{gathered}
C \propto \int_{\lambda_{a}}^{\lambda_{b}} \frac{d A(\lambda)}{d \lambda} \cdot d \lambda \quad \forall \lambda \in\left[\lambda_{1} ; \lambda_{1}\right] \text { and } \\
\lambda_{1}<\lambda_{a}<\lambda_{b}<\lambda_{2}
\end{gathered}
$$

\section{Method}

A stock solution of propranolol in purified water was first diluted to prepare the following dilutions set: 10, 20, $50,80,100$, and $120 \mu \mathrm{g} / \mathrm{mL}$. A first course of absorption measurements was carried out for all solutions. The set was then spiked with a powder of excipients so that all the suspensions of the set had an excipient concentration of $0.3 \mathrm{mg} / \mathrm{mL}$. A second course of absorption measurements was carried out for all suspensions. Finally, suspensions containing $0.1-1.0 \mathrm{mg} / \mathrm{mL}$ excipients were prepared using the previous propranolol solution $(100 \mu \mathrm{g} / \mathrm{mL})$ spiked with the excipient powder.

The baseline and blank were measured with purified water. The absorption spectra were acquired from 200 to $400 \mathrm{~nm}$ with a measurement interval of $0.50 \mathrm{~nm}$ and a measurement speed of $60 \mathrm{~nm} / \mathrm{min}$. For the "absorption method," the amplitude of the UV absorption of the sample was extracted from the spectrum at $290 \mathrm{~nm}$, whereas for the "derivative-based method," the AUC of D1 was calculated from $290 \mathrm{~nm}$ and $317 \mathrm{~nm}$.

The calibration curves for the two quantification methods with and without excipients were calculated and compared.

For the derivative-based method, the quantification response of the API spiked with excipients was correlated to the quantification response of the API in solution without excipients. 


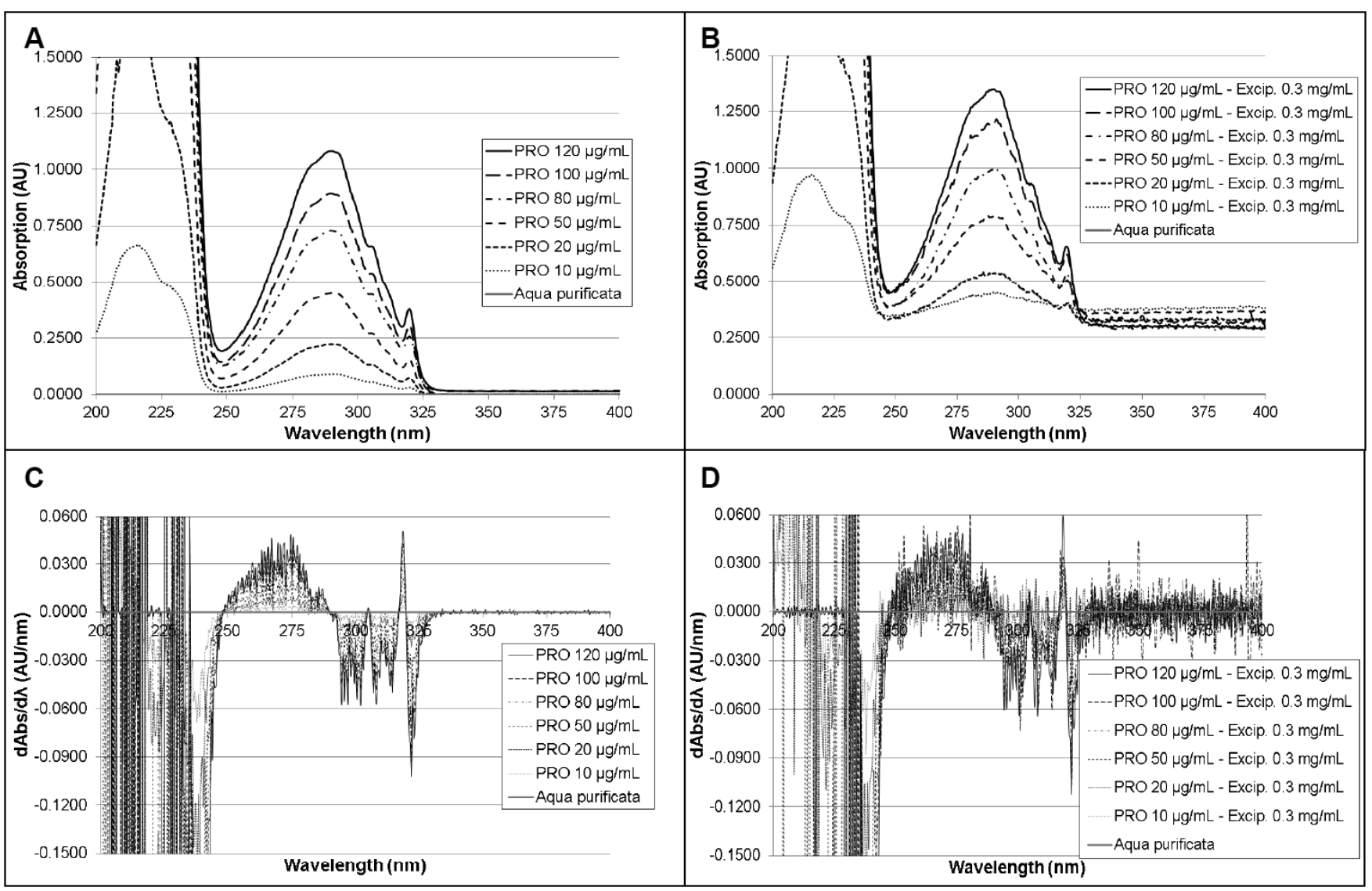

Figure 2. UV absorption and first derivative absorption spectra for propranolol (PRO) in purified water with vs. without excipients (Excip.); (A) absorption spectrum without excipients; (B) absorption spectrum with excipients. Whereas APIs and excipients are released from the drug delivery device over the dissolution test, absorptions spectra shift upward from (A) to (B). (C) First derivative absorption spectrum without excipients; (D) first derivative absorption spectrum with excipients.

Finally, the recoveries of the suspensions containing different amounts of excipients were computed by the derivative-based method to the standard solution containing $100 \mu \mathrm{g} / \mathrm{mL}$ propranolol.

\section{Validation}

The UV absorption spectra and first derivative absorption spectra for propranolol with and without excipients are presented in Figure 2.

The linearity of both methods was checked over the range of $10-120 \mu \mathrm{g} / \mathrm{mL}$ propranolol, and the determination coefficients $\left(R^{2}\right)$ as well as the limits of quantification (LOQ) are summarized in Table 1. The relative standard deviation (RSD) of the absorption method does not meet the specifications for $R^{2}$ and LOQ. Hence, the principle was not applicable. On the contrary, the derivative-based method successfully fulfilled the linearity requirements in the presence of excipients.

The calibration curves of the propranolol quantification in purified water in the presence of excipients are presented for both methods in Figure 3.

The correlation of the quantification response with excipients to the quantification response without excipients has an $R^{2}$ of 0.9998 to $120 \mu \mathrm{g} / \mathrm{mL}$ propranolol for the derivative-based method (Figure 4). This proves the successful elimination of the interferences caused by excipients (specificity).

Table 1. Linearity of the Absorption and Derivative-Based Methods With and Without Excipients

\begin{tabular}{lccc}
\hline \multicolumn{1}{c}{ Method } & Excipient concentration $(\mathbf{m g} / \mathbf{m L})$ & $\boldsymbol{R}^{\mathbf{2}}$ & LOQ $(\boldsymbol{\mu g} / \mathbf{m L})$ \\
\hline Absorption & 0.0 & 0.9998 & 0.07 \\
\hline Absorption & 0.3 & n.a. & n.a. \\
\hline Derivative & 0.0 & 0.9999 & 0.05 \\
\hline Derivative & 0.3 & 0.9999 & 0.15 \\
\hline
\end{tabular}




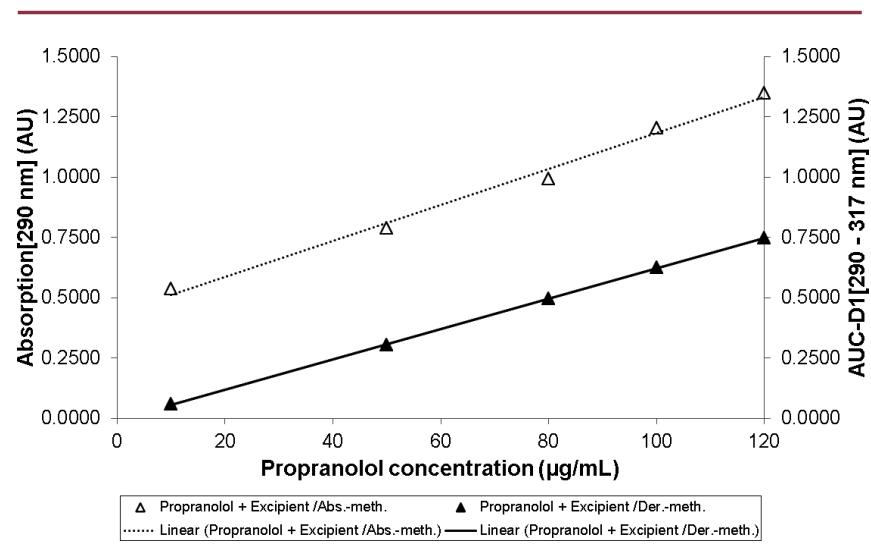

Figure 3. Linearity of the quantification methods for propranolol in purified water spiked with $0.3 \mathrm{mg} / \mathrm{mL}$ excipients; absorption at $290 \mathrm{~nm}$ and area under the curve (AUC) of the first derivative (D1) of the absorption between $290 \mathrm{~nm}$ and $317 \mathrm{~nm}$.

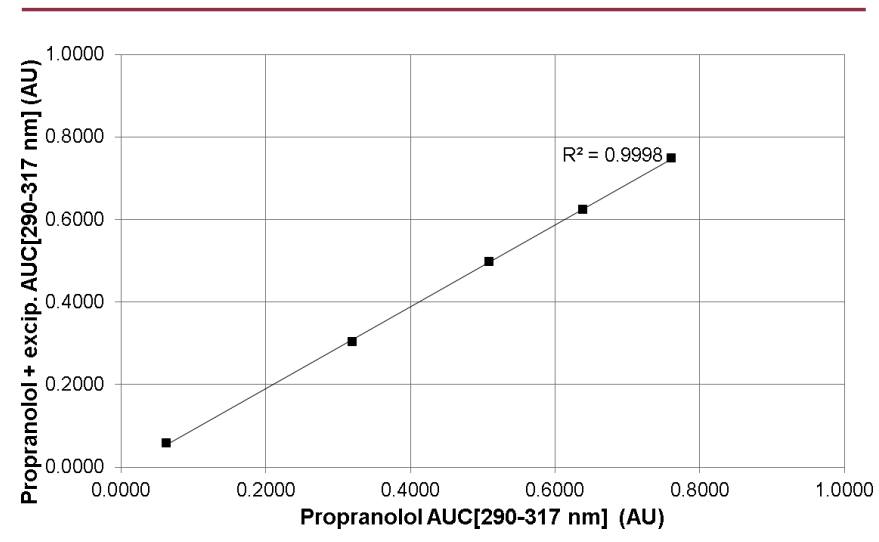

Figure 4. Correlation curve between the quantification of propranolol in purified water without and with $0.3 \mathrm{mg} / \mathrm{mL}$ excipients using the area under the curve (AUC) of the first derivative (D1) of the absorption between 290 and $317 \mathrm{~nm}$.

Finally, the recoveries of the $100 \mu \mathrm{g} / \mathrm{mL}$ propranolol solution spiked with different excipient concentrations were $96-103 \%$ for $0.1-0.7 \mathrm{mg} / \mathrm{mL}$ excipients and $94-103 \%$ for $0.1-1.0 \mathrm{mg} / \mathrm{mL}$ excipients. Hence, the accuracy of the derivative-based method was proved.

\section{CONCLUSION}

Fiber-optic systems represent a suitable option to both increase the robustness of dissolution methods and facilitate the automation for in vitro drug release testing. The time-saving effect is remarkable. Though automation by fiber-optic systems may not be applicable to all pharmaceutical products, the range of use may be broadened by the level of data processing. The most important limitation effect by matrix interferences may be overcome by mathematical solutions, such as derivative spectrophotometric methods. The use is not limited to manufacturing industry but also includes $R \& D$ projects.

\section{ACKNOWLEDGMENTS}

The authors thank the company Agilent Technologies, which kindly provided the fiber-optic system.

\section{REFERENCES}

1. Lu, X.; Lozano, R.; Shah, P. In-Situ Dissolution Testing Using Different UV Fiber Optic Probes and Instruments. Dissolution Technol. 2003, 10 (4), 6-15.

2. Nikita, S. A.; Prashik, D. B.; Madhuri, N. A. UV-spectrophotometric method development and validation of propranolol hydrochloride and flunarizine dihydrochloride in bulk drug and capsule dosage form. Contemp. Invest. Obs. Pharm. 2012, 1 (1), 19-23.

3. Corrêa, J. C. R.; Vianna-Soares, C. D.; Salgado, H. R. N. Development and Validation of Dissolution Test for Fluconazole Capsules by HPLC and Derivative UV Spectrophotometry. Chrom. Res. Int. [Online] 2012, 2012, Article 610427. http://www.hindawi.com/ journals/cri/2012/610427/ (accessed Apr 20, 2013).

4. Hajian, R.; Soltaninezhad, A. The Spectrophotometric Multicomponent Analysis of a Ternary Mixture of Paracetamol, Aspirin, and Caffeine by the Double Divisor-Ratio Spectra Derivative Method. J. Spectrosc. [Online] 2013, 2013, Article 405210. http://www. hindawi.com/journals/spectroscopy/2013/405210/ (accessed Apr 20, 2013).

5. Arnoudse, P. B.; Pardue, H. L. Evaluation of derivative spectra for the selective determination of drugs: quantitation of theophylline with phenobarbital and lightscattering components. J. Automat. Chem. 1986, 8 (2), 75-79.

6. Morton, R. A.; Stubbs, A. L. Photoelectric spectrophotometry applied to the analysis of mixtures, and vitamin A oils. Analyst 1946, 71 (845), 348-356. 